\title{
Tips and tricks for a safe laparoscopic pancreatoduodenectomy
}

\author{
Ioannis Triantafyllidis ${ }^{1}$, Brice Gayet ${ }^{2}$, David Fuks ${ }^{2}$ \\ ${ }^{1}$ Department of General Surgery, Konstantopoulio-Patision Hospital of Nea Ionia, Athens, Greece \\ ${ }^{2}$ Department of Digestive Oncologic and Metabolic Surgery, Institut Mutualiste Montsouris, Université Paris Descartes, Paris, France \\ Videosurgery Miniinv 2020; 15 (3): 383-390 \\ DOI: https://doi.org/10.5114/wiitm.2020.97977
}

\begin{abstract}
Minimally invasive pancreatoduodenectomy has been revolutionized during the last decades and, although not as rapidly or widely adopted, the laparoscopic approach seems to be feasible with various potential advantages compared to traditional open pancreatoduodenectomy. Laparoscopic pancreatoduodenectomy is a technically demanding procedure with a steep learning curve mainly due to the fact that the technique is not standardized. Technical details as well as tips and tricks of the operation are described. Standardization of the procedure is the cornerstone of the learning curve of minimally invasive pancreatoduodenectomy. One of the largest barriers of this complex procedure is the reconstruction phase with the creation of three separate anastomoses. A hybrid approach may help surgeons - especially during the initial phase of the learning curve - to overcome the difficulties associated with a fully laparoscopic reconstruction, while retaining the advantages of laparoscopy.
\end{abstract}

Key words: laparoscopic pancreatoduodenectomy, superior mesenteric artery, pancreatic adenocarcinoma, Whipple procedure, portal vein.

\section{Introduction}

Laparoscopic pancreatoduodenectomy (LPD) is a technically demanding procedure, and there is no consensus in the literature regarding its oncologic efficacy and safety when applied for the treatment of pancreatic cancer, although single-center studies have proven the feasibility and benefits of laparoscopic pancreatic surgery [1, 2].

LPD has seen much slower adoption than other laparoscopic procedures. Nevertheless, a minimally invasive pancreatoduodenectomy has the potential to reduce the inflammatory response, enhance recovery, decrease postoperative pain, morbidity and in-hospital stay, improve the quality of life and hence facilitate the implementation of adjuvant therapy.

We present our standardized laparoscopic approach with several useful "tips and tricks" to overcome various pitfalls.

\section{Operating room set-up and positioning of trocars}

The patient under general anesthesia is placed in the supine lithotomy position, with the legs abducted (French position). Pneumoperitoneum is established through a $12 \mathrm{~mm}$ optical periumbilical trocar, where a $30^{\circ}$ laparoscope is introduced, and is usually maintained at $12 \mathrm{~mm} \mathrm{Hg}$. If the distance between the xiphoid process and umbilicus is short, the first trocar should be placed $2-3 \mathrm{~cm}$ below the umbilicus. Under direct vision, five additional ports are placed: one 12 -mm port at the midpoint between the xiphoid process and the umbilicus for the laparoscopic ultrasound device and/or endoscopic stapler, and four 5-mm trocars - two working channels in the paraumbilical/pararectal region on either side of the midline and two for exposure and lateral traction as needed (Photo 1).

\section{Address for correspondence}

David Fuks MD, PhD, Department of Digestive Oncologic and Metabolic Surgery, Institut Mutualiste Montsouris,

Université Paris Descartes, 42 Boulevard Jourdan, 75014 Paris, France, e-mail: david.fuks@imm.fr 
Placing the patient in the reverse Trendelenburg position facilitates better exposure of the main anatomical structures. Furthermore, a braided silk 0 transfixing stitch piercing the round ligament, inserted subxiphoidally, provides efficient suspension of the liver (Photo 2).

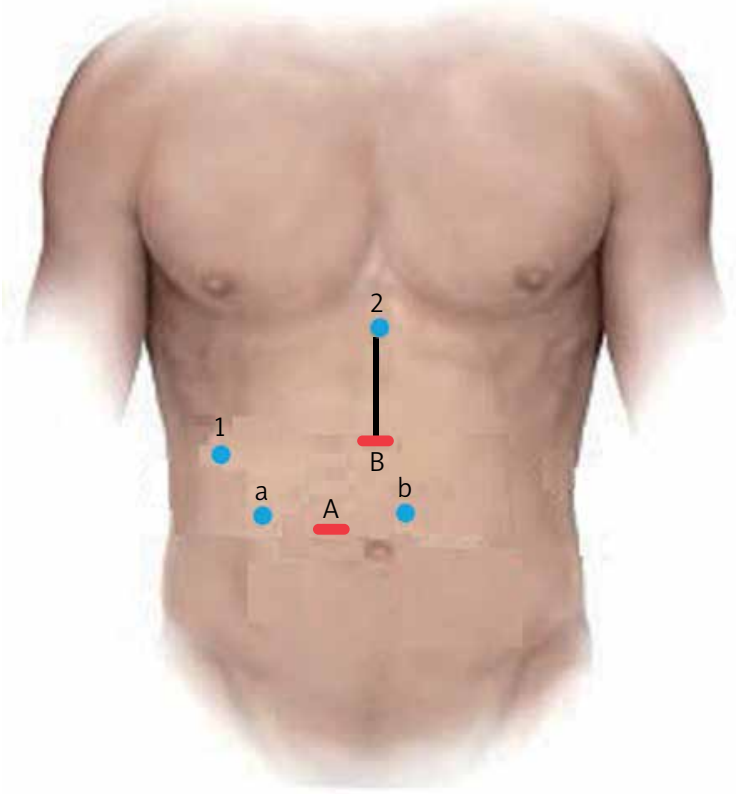

Photo 1. Trocar positioning during laparoscopic pancreatoduodenectomy. A + B: 12-mm trocar used for laparoscope/stapler insertion. a, b: 5-mm working trocars. 1, 2: 5-mm trocar exposure trocars. Black line: mini-laparotomy for the specimen's extraction and reconstruction (hybrid technique)

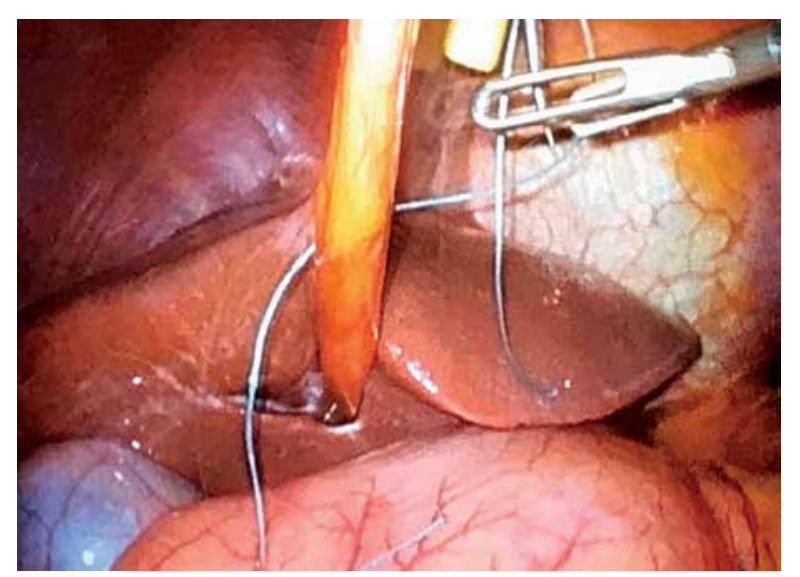

Photo 2. A braided silk 0 transfixing stitch piercing the round ligament, inserted subxiphoidally with a suture passer, provides efficient suspension of the liver
The following instruments should be available: three fenestrated bowel graspers, a firm grasper, a Maryland dissector, a short right-angle dissector, a curved needle holder, an ultrasonic dissector or the Thunderbeat device (Olympus Co, Tokyo, Japan), bipolar forceps (MicroFrance CEV134, Medtronic, Minneapolis, MN), a pair of scissors, Goldfinger, clip applier, suction and irrigation device and endobag.

\section{Intraoperative assessment of tumor resectability}

After port placement a general inspection of the peritoneal cavity is performed in order to rule out any peritoneal or liver metastases. Any suspicious peritoneal or liver nodule as well as lymph nodes at the left side of the superior mesenteric artery (SMA) and/or the aortocaval space are sent for frozen section (Photo 3) [3]. Intraoperative ultrasound is used to assess the tumor margins and its relationship with the major vessels and surrounding structures to confirm the final resectability of the tumor [4].

\section{Cholecystectomy}

The first step of the operation is a laparoscopic cholecystectomy. Any adhesions are taken down with ultrasonic shears and/or scissors. The cystic duct and cystic artery are divided, and the gallbladder is removed using an extraction bag.

\section{Dissection of the right colon and duodenum}

The gastrocolic ligament is opened to enter the lesser sac and expose the retroperitoneal area and ultimately the pancreas. Division of the gastrocolic ligament is performed below the gastroepiploic vessels. Subsequently, under appropriate cephalad traction by grasping the stomach antrum or body with atraumatic forceps, any adhesions present between the posterior surface of the stomach and the anterior surface of the pancreas are taken down, leading to direct visualization of the pancreas. We continue dissection laterally to fully mobilize the hepatic flexure and expose the duodenum, while lymphadenectomy of the infrapyloric station is completed (no. 6). Right colon mobilization facilitates easier mobilization of the duodenum and control of the gastrocolic 

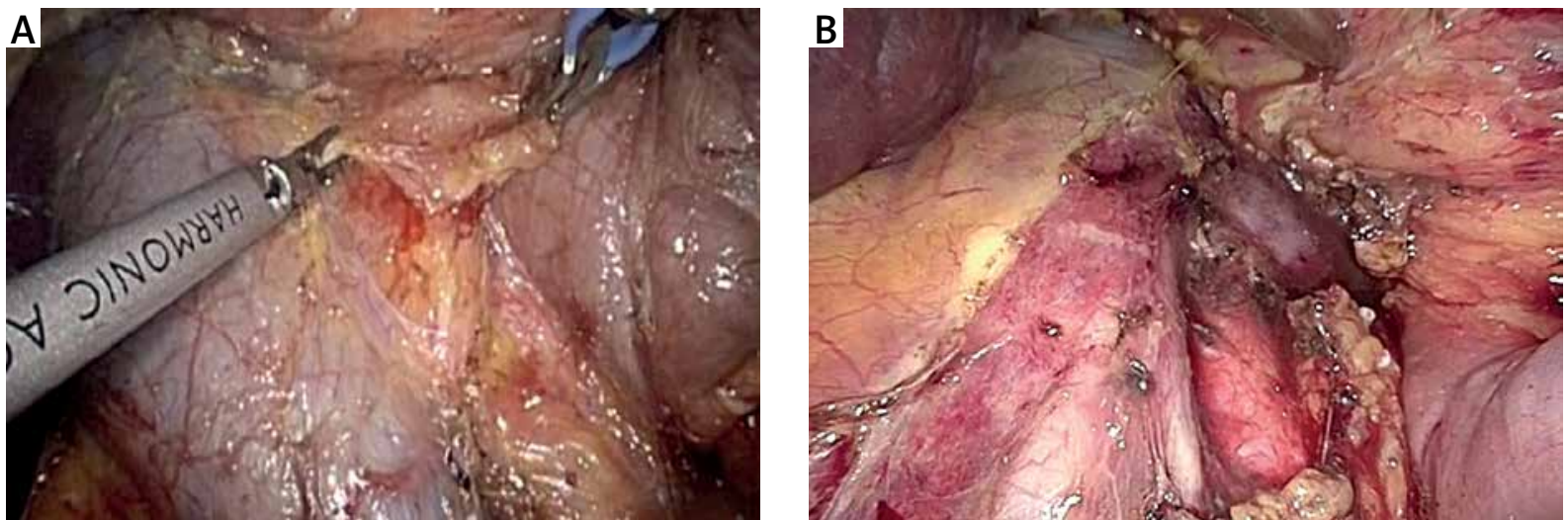

Photo 3. Aortocaval dissection. A wide Kocher maneuver allows excellent exposure of the inferior vena cava, aortocaval space and right celiac plexus dissection (lymph node 16b1). Any suspicious peritoneal or liver nodule as well as lymph nodes at the left side of the superior mesenteric artery (SMA) and/or the aortocaval space are sent for frozen section

trunk of Henle as well as of the superior mesenteric vein (SMV) and first jejunal venous branch. Subsequently, an extended Kocher maneuver is performed to the level of the left renal vein, taking all the retroperitoneal attachments off the pancreas and exposing the medial and inferior borders of the SMA. The SMA is encircled with a vessel tape. Dissection along the SMA facilitates the identification of the inferior pancreatoduodenal artery. During the Kocher maneuver, we ensure gentle traction on the duodenum in order to avoid an inadvertent perforation. A wide Kocher maneuver allows excellent exposure of the inferior vena cava, aortocaval space and right celiac plexus dissection (lymph station no. 16b1) (Photo 3). Although inter-aorto-caval lymphadenectomy remains controversial, we believe that it is safe and provides better staging of pancreatic cancer and thus is reasonable.

\section{Dissection of the portal venous confluence}

The peritoneum overlying the inferior border of the pancreas is opened and dissected to identify the infrapancreatic part of the SMV. Blunt dissection is carried on along the anterior surface of the SMV, progressively separating the vein from the posterior aspect of the pancreatic neck and eventually leading to the identification of the spleno-mesenteric confluence. Ligation of the gastrocolic trunk of Henle is mandatory before beginning the creation of the retropancreatic "tunnel" from below. During this step, the laparoscopic approach offers a significant advantage represented by the magnified visualiza- tion of the "tunnel". Injury of the porto-mesenteric veins should be avoided at all costs by very gentle preparation.

\section{Dissection of the hepatoduodenal ligament}

We now proceed to the dissection of the common and proper hepatic artery. This step ensures proper recognition of the gastroduodenal artery (GDA) before its transection (Photo 4). An adequate lymph node clearance of the hepatoduodenal ligament is performed (no. 12). Grasping or any traction on the GDA should be avoided at all costs due to the fragility of the vessel. Additionally, care should be taken to avoid any avulsion to the superior anterior pancreaticoduodenal artery. Above all, a hepatic artery injury should be avoided during this step by using scissors for dissection. The common hepatic artery is lifted by a vessel tape to facilitate the dissection of surrounding lymph nodes. In some cases, an aberrant left hepatic artery arises from the left gastric artery and runs within the lesser sac. This vessel should be preserved at all costs and used in case of unexpected potential complications requiring occlusion of the common hepatic artery (i.e. bleeding from the GDA stump). We then identify the common bile duct (CBD) and all lymphatic tissue lateral and posterior to it is cleared inferiorly towards the pancreas. The surgeon should be aware of the presence of a replaced or aberrant right hepatic artery which will be identified posterior to the CBD. The CBD is transected with cold scissors and 

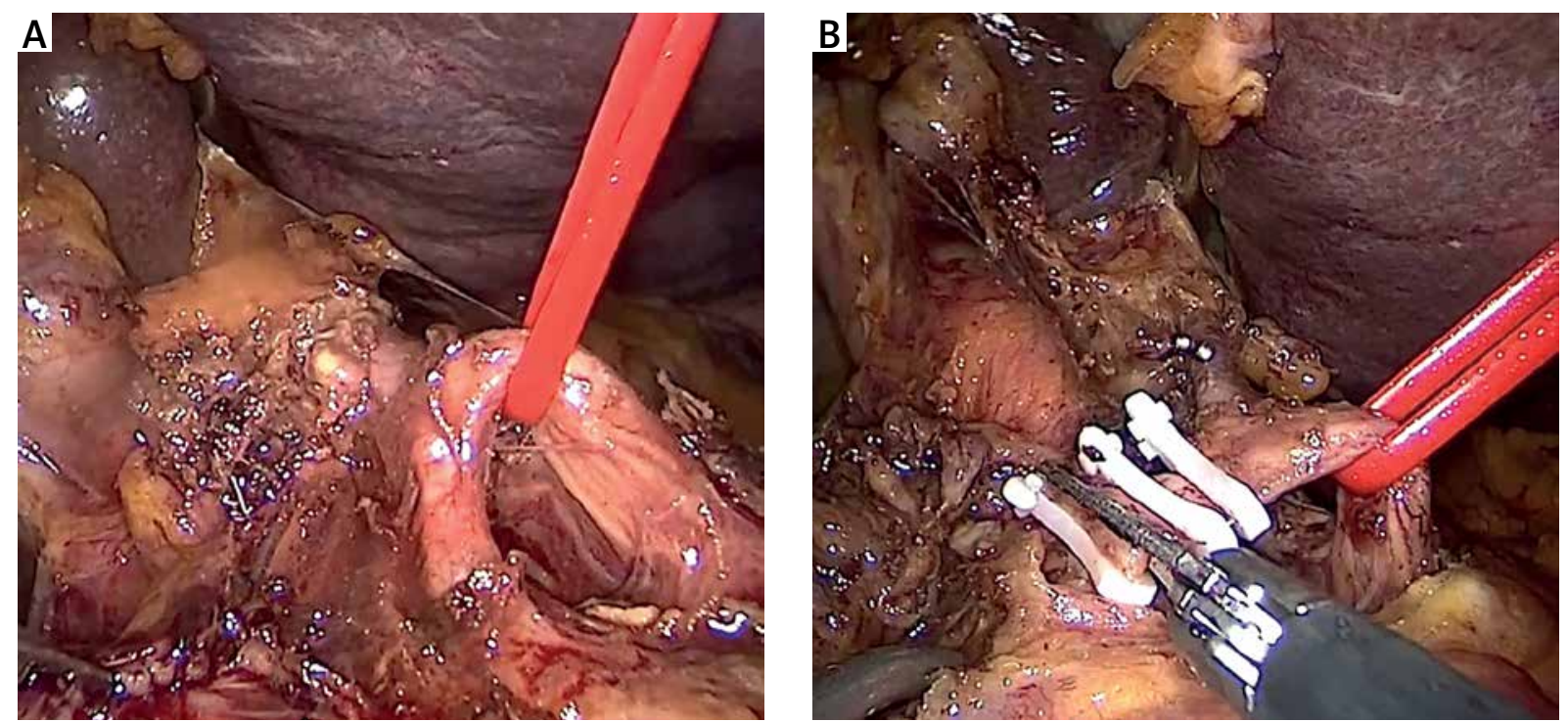

Photo 4. The gastroduodenal artery (GDA) is ligated with clips and/or stiches, near its origin from the common hepatic artery just above the superior margin of the pancreas. This step ensures proper recognition of the GDA before its transection. Grasping or any traction on the GDA should be avoided at all costs due to

the fragility of the vessel

an endo-bulldog clamp should be placed to secure its proximal aspect, to avoid bile spillage. "Cold" transection preserves a normal bile duct wall for the subsequent bilio-enteric anastomosis. Usually, the CBD is transected $2-3 \mathrm{~cm}$ cephalad to the superior edge of the pancreas, unless we are dealing with a mid-distal cholangiocarcinoma. In such cases, resection of the extrahepatic biliary tree with bilio-enteric anastomosis in the hilum is mandatory. In some cases associated with severe cholangitis, indocyanine green (ICG) technology will allow easy identification of the CBD.

\section{Transection of the jejunum and the stomach}

The next step is stomach and jejunal transection. The ligament of Treitz is identified, mobilized from its retroperitoneal attachments and divided, allowing the proximal jejunum to be pulled underneath the SMA. The jejunum is transected with a linear endoscopic stapler, approximately $15 \mathrm{~cm}$ distal to the Treitz ligament, and the mesentery is divided with an energy device at the border of the mesentery and serosa. The transection of the jejunal mesentery is continued up to the uncinate process and the SMV. We then divide the greater omentum at the level of the antrum and the right gastroepiploic artery is divided using bipolar diathermy or an energy device. The lesser omentum is opened and the stomach is transected with an endoscopic stapler just proximal to the pylorus. Alternatively to a Whipple pancreatoduodenectomy [5], a pylorus-preserving pancreatoduodenectomy [6] may be performed and in such a case the duodenum is transected $2-3 \mathrm{~cm}$ distal to the pylorus, in order to preserve its function.

\section{Ligation of the gastroduodenal artery and completion of the retropancreatic "tunnel"}

At this step, the GDA is ligated with clips and/ or stiches, near its origin from the common hepatic artery just above the superior margin of the pancreas (Photo 4). Prior to GDA ligation, common hepatic artery flow is confirmed on Doppler after occlusion of the GDA using an endo-bulldog. The supra-pancreatic portal vein (PV) is identified at the apex of the triangle formed by the common hepatic artery, GDA, and the superior border of the pancreas. The avascular plane between the posterior aspect of the pancreatic neck and the PV is developed in a cephalad-to-caudal direction, thereby completing the retro-pancreatic "tunnel" from above (Photo 5). 


\section{Transection of the pancreas and retroperitoneal dissection}

We can now proceed to pancreatic transection along the previously created "tunnel" with an energy device, laparoscopic linear stapler or with electro-cautery. However, it seems important to perform a "cold" transection of the pancreatic duct with scissors, 2-3 $\mathrm{mm}$ from the right side of the parenchymal transection line. This enables an easy future passage of the pancreatic juice. The pancreatic remnant is mobilized 2-3 cm to the tail, if a pancreaticojejunostomy is planned or furthermore (up to $4-5 \mathrm{~cm}$ ) to the groove of the splenic artery on the posterior surface of the pancreas if a pancreatogastrostomy will be performed, and covered with gauze in order to prevent spillage of the pancreatic juice into the peritoneal cavity. When necessary, a frozen section of the transected section of the pancreas is performed.

The inferior pancreaticoduodenal arcade is controlled, and thereafter both the duodenum and the first jejunal loop can easily be rotated under the mesenteric vessels. Pulling the jejunum underneath the ligament of Treitz - and not through a defect in the transverse mesocolon - avoids jejunal twisting that can be easily overlooked laparoscopically and provides a tension-free loop for reconstruction. The dissection continues towards the porto-mesenteric confluence by pulling the specimen upwards and to the right while dissecting and ligating all short vessels to the uncinate process. The uncinate process can be dissected free from the SMA using an energy device; however, occasionally it will require clips or ligature. We now transect the retroperitoneum along

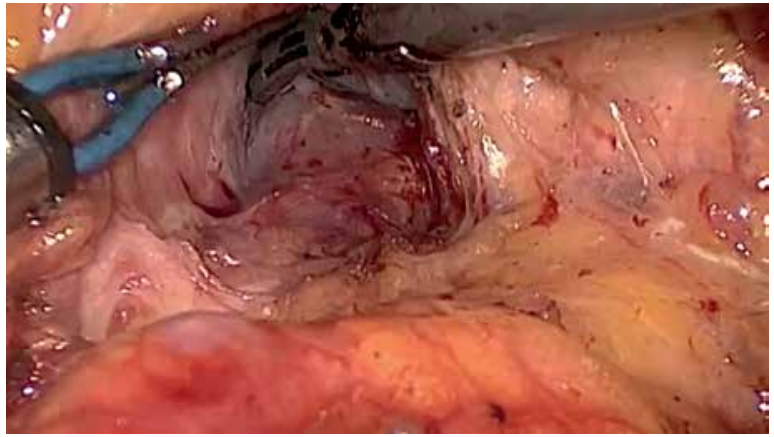

Photo 5. The avascular plane between the posterior aspect of the pancreatic neck and the portal vein is developed in a cephalad-to-caudal direction, thereby completing the retro-pancreatic "tunnel" from above

the SMA (Photo 6). The surgeon should pay attention to the small mesenteric tributaries to the uncinate process as they can easily be avulsed, resulting in troublesome hemorrhage. Cephalad, we encounter and individually ligate the superior pancreaticoduodenal arcade. With the dissection along the superior mesenteric vessels, the mobilization of the specimen is completed (Photo 7). The extraction of the specimen is performed using a laparoscopic endobag, through a mini-laparotomy extended among the $5 \mathrm{~mm}$ and $12 \mathrm{~mm}$ trocars in the midline (Photo 1) and after inserting a plastic wound protector.

\section{Vascular resection}

Resection should be performed either in a wedge or cylindrical manner using cold scissors. Although vascular clamping is prolonged compared to open
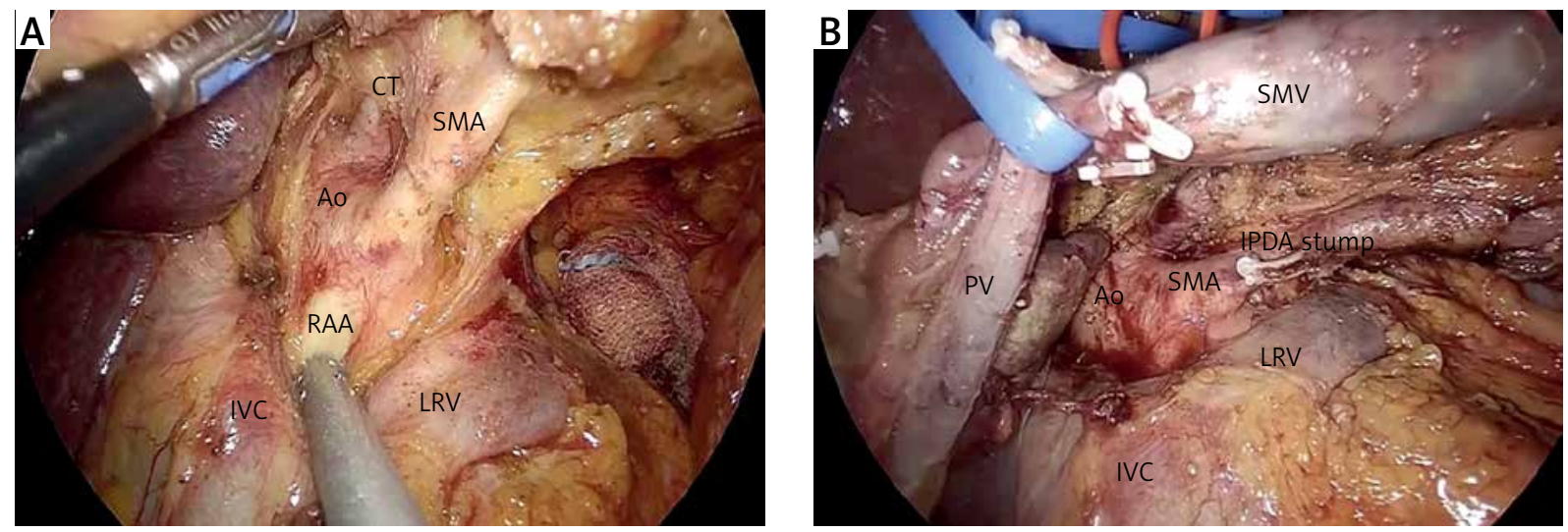

Photo 6. The uncinate process can be dissected free from the superior mesenteric artery (SMA) using an energy device; however, occasionally it will require clips or ligature. With the dissection along the superior mesenteric vessels, the mobilization of the specimen is completed 


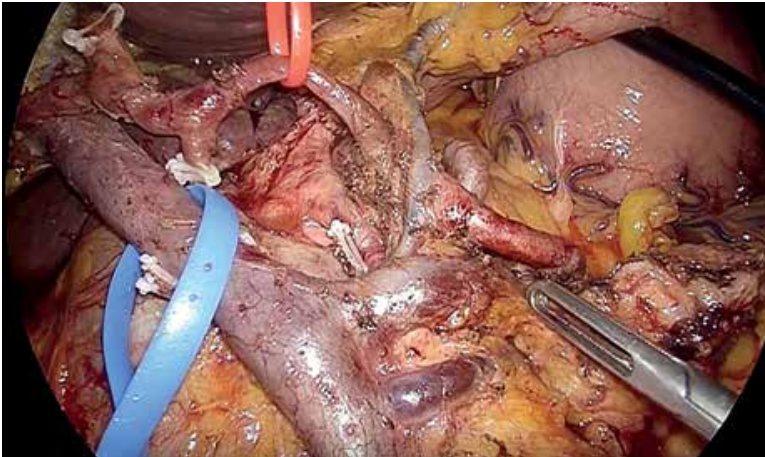

Photo 7. Endoscopic view of the major vasculature of the surgical field after the specimen's complete mobilization

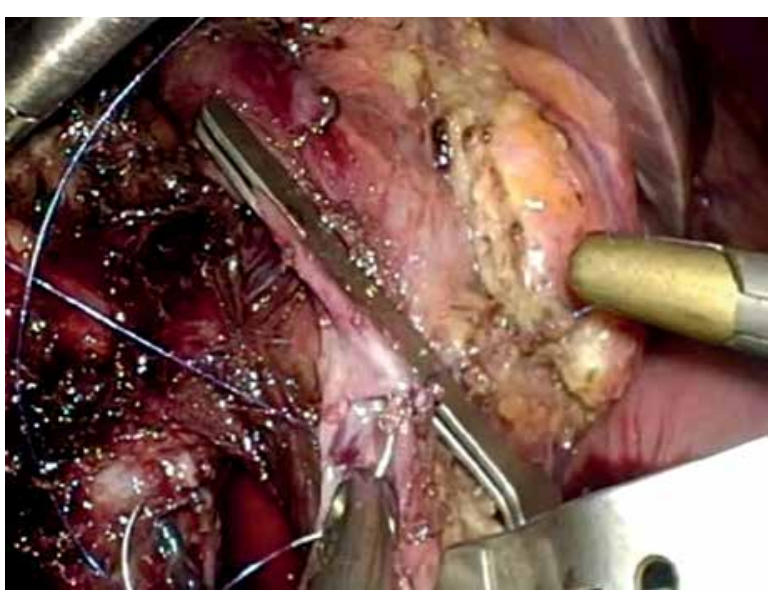

Photo 8. Superior mesenteric and splenic veins are controlled with vascular clamps. After resection of the invaded venous segment, the distance between the cut edges is measured. Reconstruction may be carried out with a monofilament continuous suture (e.g. Prolene $4 / 0$ or $5 / 0$ ). A defect between the proximal and distal vein edges $>4 \mathrm{~cm}$ is considered an indication for an interposition graft

pancreatoduodenectomy, it seems that clamping is better tolerated during the laparoscopic approach due to less mobilization and twisting of the intestine. Extensive mobilization of the porto-mesenteric and splenic confluence is mandatory. Thereafter, portal, superior mesenteric and splenic veins are controlled with vascular clamps. Reconstruction may be carried out with a monofilament continuous suture (e.g. Prolene 4/0 or 5/0) (Photo 8). A defect between the proximal and distal vein edges $>4 \mathrm{~cm}$ is considered an indication for interposition graft. After resection of the invaded venous segment, the distance between the cut edges is measured. A prosthetic graft is prepared based on this measurement. Reconstruction is performed in a caudal to cephalic manner. Caudal anastomosis is carried out after the graft and the vein are rotated $360^{\circ}$ with a monofilament running suture while the cephalad anastomosis is performed from the posterior wall and then shifted to the anterior wall with a continuous suture as well. The graft is flushed with heparin solution after the reconstruction is completed. Postoperative thromboprophylaxis (with low molecular weight heparin initially and thereafter with warfarin) is of paramount importance for the graft patency [7].

\section{Reconstruction}

The reconstruction phase is carried out either intracorporeally or through the mini-laparotomy performed for the specimen's extraction and encompasses the performance of a pancreaticojejunostomy, hepaticojejunostomy and a gastrojejunostomy or duodenojejunostomy. It is advisable to create the anastomoses at the beginning of the learning curve via a mini-laparotomy. Management of the pancreatic stump is the real key point of the entire procedure.

For the pancreatojejunostomy, we perform a twolayer end-to-side duct-to-mucosa anastomosis in all cases. The free end of a $20-30 \mathrm{~cm}$ jejunum loop is brought into proximity with the pancreatic stump in a retro-mesocolic fashion. It is necessary to check the position of the jejunum's mesentery to avoid any torsion. The anastomosis begins with the construction of the posterior trans-pancreatic/ seromuscular anastomotic row, which is fashioned using a single-layer synthetic absorbable monofilament suture (polydioxanone 4/0). The jejunum loop is stitched $4-6 \mathrm{~cm}$ distal from the stump in the middle of the posterior semi-circle. The pancreatic stump is stitched approximately $1.5-2.0 \mathrm{~cm}$ distal to the cut edge depending on the texture and the size of the remnant. The distance between the sutures is $0.5-1.0 \mathrm{~cm}$. Electrocautery or a harmonic scalpel is used to create a small $(2-3 \mathrm{~mm})$ enterotomy in the jejunum (Photo 9). Thereafter, a duct-to-mucosa pancreaticojejunostomy is created using a synthetic absorbable monofilament suture (polydioxanone 5/0) in an interrupted fashion. Six to eight sutures are usually placed, depending on duct size. Implementation of a pancreatic stent is not mandatory in our opinion. However, trans-anastomotic stenting, 

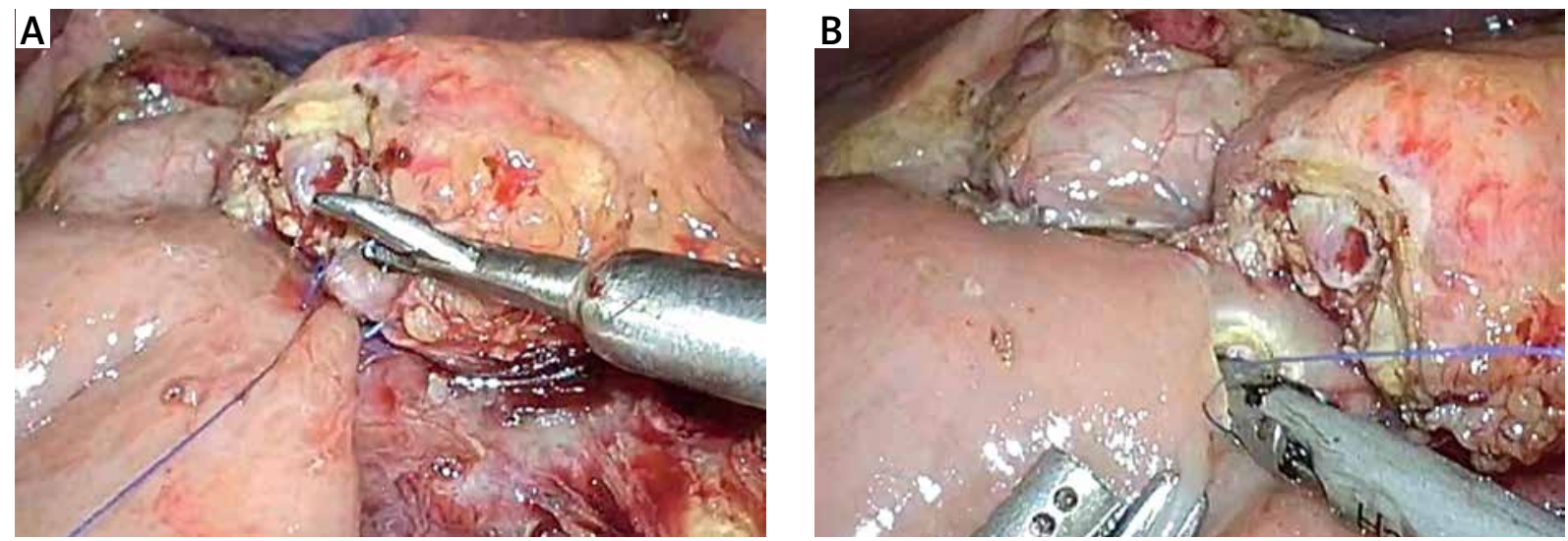

Photo 9. For the pancreatojejunostomy, we perform a two-layer end-to-side duct-to-mucosa anastomosis. The anastomosis begins with the construction of the posterior trans-pancreatic/seromuscular anastomotic row, which is fashioned using a single-layer synthetic absorbable monofilament suture (polydioxanone 4/0). The jejunum loop is stitched 4-6 cm distal from the stump in the middle of the posterior semi-circle. The pancreatic stump is stitched approximately $1.5-2.0 \mathrm{~cm}$ distal to the cut edge depending on the texture and the size of the remnant. The distance between the sutures is $0.5-1.0 \mathrm{~cm}$. Thereafter, a duct-to-mucosa pancreaticojejunostomy is created using a synthetic absorbable monofilament suture (polydioxanone 5/0) in an interrupted fashion. Six to eight sutures are usually placed, depending on the duct size

either internal or external, still remains a controversial issue in pancreatic surgery. The anterior row is performed in the same manner as the posterior one (parallel to the posterior row of the pancreas). However, in cases of a soft and fragile pancreatic remnant or a small pancreatic duct we prefer a triple purse-string telescoped pancreatogastrostomy, usually via the mini-laparotomy [8]. In such cases the reconstruction begins with the bilio-enteric anastomosis and thereafter the pancreatogastrostomy and finally gastrojejunostomy is performed.

The completion of a pancreaticojejunostomy is followed by the construction of an end-to-side bilio-enteric anastomosis using interrupted 4/0 synthetic absorbable monofilament sutures in cases of a narrow $(<5 \mathrm{~mm})$ bile duct. The posterior row of the anastomosis is fashioned first and usually requires three to four interrupted sutures. Subsequently, the anterior row of the hepaticojejunostomy is constructed in a similar manner. Alternatively, the anastomosis is performed with two running semicircular sutures $2 \mathrm{~mm}$ apart, in a clockwise fashion beginning at 9 and 3 o'clock, respectively. The incision of the jejunum wall is performed $10-15 \mathrm{~cm}$ distal to the pancreaticojejunostomy, using electrocautery or a harmonic scalpel. In order to minimize the tension of the bilio-enteric anastomosis, it is advisable to anchor the free end of the jejunal loop to the hilar plate using one or two interrupted stitches.
The gastrojejunostomy or duodenojejunostomy is the next and final reconstructive step. In both options the jejunum loop is pulled up in an antecolic position and an anastomosis is performed $30-40 \mathrm{~cm}$ below the hepaticojejunostomy. The gastrojejunostomy is performed with a stapling technique in the lowest part of the gastric stump on the greater curvature and sutured closure of the common enterotomy is done in two layers. Alternatively, a "hand-sewn" gastrojejunostomy or end-to-side duodenojejunostomy in a one- or two-layered fashion can be formed.

Finally, the peritoneal cavity is inspected for bleeding, bile leakage, or remaining enterotomy defects. Two abdominal 19 French closed suction drains are placed close to the pancreatojejunostomy or pancreatogastrostomy through the foramen of Winslow and the lesser sac. The greater omentum is pulled up to cover the pancreaticojejunostomy so that the gastrojejunostomy or duodenojejunostomy is not in direct contact with the pancreaticojejunostomy and is fixed in this position with clips or stiches. The falciform ligament is used to create a flap to cover the stump of the GDA, in order to prevent erosion in case of pancreatic leakage. The flap may be marked with a metallic clip for recognition if a postoperative angiography is needed. Abdominal wall fascial defects $>5 \mathrm{~mm}$ are closed in the usual manner. 


\section{Conclusions}

Standardization of the procedure is the cornerstone of the learning curve of minimally invasive pancreatoduodenectomy. One of the main hurdles of this complex procedure is the reconstruction phase with the creation of three separate anastomoses. A hybrid approach may help surgeons - especially during the initial phase of the learning curve - to overcome the difficulties associated with a fully laparoscopic reconstruction, while retaining the advantages of laparoscopy.

\section{Conflict of interest}

The authors declare no conflict of interest.

\section{References}

1. Edwin B, Sahakyan MA, Abu Hilal M, et al.; EAES Consensus Conference Study Group. Laparoscopic surgery for pancreatic neoplasms: the European association for endoscopic surgery clinical consensus conference. Surg Endosc 2017; 31: 2023-41.

2. Gagner M, Pomp A. Laparoscopic pylorus-preserving pancreatoduodenectomy. Surg Endosc 1994; 8: 408-10.

3. Azagra JS, Arru L, Estévez S, et al. Laparoscopic pancreatoduodenectomy with initial approach to the superior mesenteric artery. Videosurgery Miniinv 2015; 10: 450-7.

4. Corcione F, Pirozzi F, Cuccurullo D, et al. Laparoscopic pancreatoduodenectomy: experience of 22 cases. Surg Endosc 2013; 27: 2131-6.

5. Whipple AO, Parsons WB, Mullins CR. Treatment of carcinoma of the ampulla of Vater. Ann Surg 1935; 102: 763-9.

6. Whipple AO. The rationale of radical surgery for cancer of the pancreas andampullary region. Ann Surg 1941; 114: 612-5.

7. Wang X, Cai Y, Zhao W, et al. Laparoscopic pancreatoduodenectomy combined with portal-superior mesenteric vein resection and reconstruction with interposition graft: case series. Medicine 2019; 98: e14204.

8. Addeo P, Rosso E, Fuchshuber P, et al. Double purse-string telescoped pancreaticogastrostomy: an expedient, safe, and easy technique. J Am Coll Surg 2013; 216: e27-33.

Received: 16.07 .2020 , accepted: 28.07 .2020 